\title{
Correspondence
}

\section{Intra-articular volume as an important factor governing macromolecular half life in synovial fluid}

SIR. We read with interest the paper by Dr Page-Thomas and colleagues entitled Clearance of proteoglycan from joint cavities'.' A striking finding was that the half life of proteoglycan aggregates in the joint cavity was unaltered by synovitis. It would be wrong to conclude that the proteoglycan clearance rate was unaffected, however. As pointed out by Simkin and others intra-articular fluid volume must be taken into consideration as well as half life when interpreting clearance studies. ${ }^{2}$ When both variables were considered Wallis et al observed a faster clearance rate for radiolabelled albumin from rheumatoid joints than from osteoarticular joints. ${ }^{2}$

The importance of intra-articular volume arises because most mechanisms for removal of molecules from joints (whether by diffusion, convection, or degradation) depend on concentration. ${ }^{3}$ This results in a monoexponential shaped removal curve as described in Dr Page-Thomas's paper. Monoexponential curves are in keeping with other studies reflecting first order kinetics. ${ }^{2}$ Such kinetics allow the removal of proteoglycan to be expressed as a single half life (time taken for the knee radioactivity to diminish to $50 \%$ of the original value) or its close relation, the removal rate constant (the fraction of the original amount of injected proteoglycan that is removed in unit time, expressed as min ').

Concentration depends on the volume of synovial fluid in which the injected radiolabelled proteoglycan was distributed. as well as the mass injected. In the study described it would be anticipated that the inflamed rabbit joints contained greater volumes of synovial fluid than the control joints. If this is correct then the mechanism removing proteoglycan from the arthritic joint must have been enhanced, as it achieved a similar half life despite operating at a lower concentration of proteoglycan. To put it another way, it is likely that the clearance rate (volume of fluid cleared of proteoglycan in unit time, expressed in $\mathrm{ml} / \mathrm{min}$ ) was considerably larger in the inflamed joints.

The above argument can be set out more rigorously as follows: Let $-\mathrm{dm} / \mathrm{dt}$ be rate of removal of test solute at time $t$. after injection of initial test mass $m_{0}$ at time zero into volume $\mathrm{V}$. At time the concentration $\mathrm{C}$ of the solute in the synovial fluid will depend on the mass $\mathrm{m}$ remaining

$$
\mathrm{C}=\mathrm{m} / \mathrm{V}
$$

The expression defining clearance rate, $\gamma$, of a solute is

$$
\gamma=(-\mathrm{dm} / \mathrm{dt}) / \mathrm{C}
$$

As $\mathrm{C}=\mathrm{m} / \mathrm{V}$, then

$$
-\mathrm{dm} / \mathrm{dt}=\gamma(\mathrm{m} / \mathrm{V})
$$

On integration, this becomes

$$
\log _{c} m=-\gamma(t / V)+\log _{c} m_{1}
$$

The removal rate constant is the slope of the $\log _{\mathrm{c}} \mathrm{m}$ versus $\mathrm{t}$ plot, which we will call $\lambda$. From the above expression the slope is $-\gamma / \mathrm{V}$, so

$$
\lambda=-\gamma / \mathrm{V}
$$

Half life, $t_{1 / 2}$, is $0 \cdot 693 / \lambda$, so

$$
t_{1 / 2}=-() \cdot 693 \mathrm{~V} / \gamma
$$

Thus the measured half life depends not only on $\gamma$, the clearance rate, but also on $\mathrm{V}$, the distribution volume of the solute.

Similarly, the clearance rate $(\gamma)$ of the proteoglycan depends not only on rate constant (or $t_{1 / 2}$ ) but also on volume ${ }^{2}$

$$
\gamma(\mathrm{ml} / \mathrm{min})=\lambda\left(\min ^{1}\right) \times \mathrm{V}(\mathrm{ml})
$$

For example normal rabbit knees contain $\sim(0 .() 24 \mathrm{ml}$ of synovial fluid, ${ }^{+}$and a moderate effusion that might be expected in a non-fibrosed knee with an intra-articular pressure of $\sim 20 \mathrm{~cm}$ water would be $\sim 2 \cdot() \mathrm{ml}$. $^{5}$ When the figures quoted by Dr Page-Thomas for injection volumes and half lives are used the clearance of proteoglycan aggregate from the inflamed knees would be $1.91 \times 10^{3} \mathrm{ml} / \mathrm{min} \mathrm{com}-$ pared with clearance from the control knees of $\left(0.38 \times 10^{3}\right.$ $\mathrm{ml} / \mathrm{min}$, a fivefold difference. Even if the effusion were only $0.4 \mathrm{ml}$ (compliance reduced by fibrosis) the arthritic clearance would still be $69 \%$ faster than the control.

Unfortunately the authors do not include data about the synovial fluid volumes in the paper and have only presented half lives not clearance rates. This could easily lead the reader to erroneous conclusions. Our interpretation of the interesting observations is that, if intra-articular volume was greater in the arthritic knee yet the half life was not significantly slower, then the clearance of proteoglycan was greater in the arthritic knee.

Department of Physiology.

JRODNEY I.EVICK

St George's Hospital Medical School.

Cranmer Terrace.

London SW17 0RE

Bone and Joint Research Unit . PAUI. WTHOMPSON The London Hospital Medical College.

Turner Street.

London E1 2AD 


\section{References}

I Page-Thomas D P. Bard D. King B. Dingle J T. Clearance of proteoglycan from joint cavities. Ann Rheum Dis 1987; 46: 934-7.

2 Wallis W J. Simkin P A. Nelp W B. Foster D M. Intraarticular volume and clearance in human synovial effusions. Arthritis Rheum 1985: 28: 441-9.

3 Levick J R. Blood flow and mass transport in synovial joints. In: Renkin E M. Michel C C. eds. Handhook of physiology. Section II. The cardiovascular svstem IV. Bethesda, USA: American Physiological Socicty, 1984: 917-47.

4 Knox P. Levick J R. McDonald J N. Synovial fluid-its mass. macromolecular content and pressure in major limb joints of the rabbit. Q J Exp Phvsiol 1988: 73: 33-45.

5 Levick J R. Synovial fluid and trans-synovial flow in stationary and moving normal joints. In: Helminen Het al, eds. Joint loading. Bristol: Wright. 1987: 149-86.

SiR, We were interested to read the comments of Drs Levick and Thompson. Although accepting their observation that as our labelled injections in the synovial fluid of arthritic joints are more dilute the apparent clearance rates are greater, we feel that they may be overinterpreting our data, and wish to make two main points.

Firstly, the experiments measure only the clearance of labelled material and do not take into account the effects of existing proteoglycans in the joint which may compete. Without conducting a detailed analysis of the composition of the synovial fluid, an important study, but one beyond the scope of our original paper, it is very difficult to make sensible predictions of the extent of this effect and to calculate overall clearance rates. We chose to express our results as half lives precisely because this measurement makes no assumptions about the absolute intra-articular concentrations of proteoglycan.

Secondly, the experimental equipment, as described in our paper, measures radioactivity over the whole joint, including the synovium. The data therefore represent clearance from the whole joint area, rather than just the joint cavity. Although one may anticipate that loss from the joint cavity is the rate limiting step, our finding that at 42 hours between a half and three quarters of the residual radioactivity was associated with the synovium suggests that this may not be the case for the whole duration of the experiment. Further detailed experiments are clearly necessary to establish tissue distributions at earlier times, whether or not the radioactivity in the synovium is intracellular or extracellular, and whether it is located predominantly on the surface of the tissue or distributed throughout its volume.

In conclusion, we do not dispute the physical calculations, but question their direct application to competing clearance mechanisms across anatomical structures of complex and variable composition. This is not to denigrate the observations of Dr Levick and his associates, merely to point out that any simple mathematical model of synovial clearance has limitations.

Strangeways Research Laboratory, Worts Causeway,

Cambridge CB1 4RN

\section{Anticardiolipin antibodies, livedo reticularis, and cerebrovascular accidents in SLE}

SIR, We read with interest the recent article by McHughost al concerning the incidence of major cerebrovascufar events and livedo reticularis in three patients with systempe lupus erythematosus (SLE) and anticardiolipin antibodies. ${ }^{1}$ We have encountered a similar association in out patients. Of the 35 patients with cerebrovascular disease and anticardiolipin antibodies seen in our clinics, only o $\overline{\bar{B}}$. has suffered from subarachnoid haemorrhage, and this resulted from a ruptured aneurysm. A significant numbet were also hypertensive, however, and we therefore question whether hypertension could have been a factor $\mathbb{T}$ their single patient with cerebral haemhorrhage.

The authors have advocated treatment of the cerebra thromboses with immunosuppression (pulse methylpredn $\overrightarrow{1-}$ solone and cyclophosphamide). The prophylactic efficaey of this regimen in the long term prevention of further thrombotic events is questionable if an antibody mediate mechanism for thrombosis is being proposed. Antibot levels rise fairly rapidly on completion of the pulge therapy, and these levels may not even be fully suppressed. Furthermore, we have encountered several patients wiff cerebral thromboses and anticardiolipin antibodies wh have had no other evidence of lupus activity eith clinically or serologically. We have advised anticoagulation for prophylaxis and are currently undertaking prospectioe trials in order to assess its efficacy. It certainly would avoli the hazards of long term immunosuppression, which wouबie otherwise not be indicated for control and treatment of $\mathrm{t}$ 征 underlying disease process.

The Lupus Arthritis Research Unit,

The Rayne Institute,

St Thomas's Hospital,

London SE1 7EH

\section{References}

1 McHugh N J, Maymo J, Skinner R P. James I, Maddison PQ Anticardiolipin antibodies, livedo reticularis, and major carcbrovascular and renal disease in systemic lupus erythematosto Ann Rheum Dis 1988: 47: 110-5.

2 Asherson R A. Mayou S. Black M. et al. Livedo reticularn connective tissue disease, anticardiolipin antibodies and CAS complications [Abstract]. Arthritis Rheum 1987; 30: S69. N

3 Harris E N, Gharavi A E, Asherson R A. Hughes G R R Cerebral infarction in systemic lupus erythematosus: association with anticardiolipin antibodies. Clin Exp Rheumatol 1984:F्: 47-51.

4 Asherson R A, Baguley E, Khamashta M, et al. The spectrum cerebrovascular disease and antiphospholipid antibodies. Semin Arthritis Rheum (in press).

SIR, I am grateful for the interest shown in our paper by ff Baguley and her colleagues, though their letter contaiß several inaccuracies. We reported four of 98 patients wi画 systemic lupus erythematosus suffering cerebrovascular
B KING

J T DINGLE 